\title{
DIAGNÓSTICOS DE ENFERMAGEM DE IDOSAS CARENTES DE UM PROGRAMA DE SAÚDE DA FAMÍLIA (PSF)a
}

\author{
Nursing Diagnoses of Pauper Elderly Women of a \\ Family Health Program (FHP) \\ Diagnósticos de Enfermería de Ancianas Carentes \\ de un Programa de Salud de la Familia (PSF)
}

Maria José Sanches Marin ${ }^{1}$

Fabiana Aroni Ricci ${ }^{4}$

\author{
Luiz Carlos de Oliveira Cecílio 2
}

Suelaine Druzian ${ }^{5}$
Luciane Cristine Ribeiro Rodrigues ${ }^{3}$

\section{Resumo}

Considerando que as mulheres idosas e pobres são propensas à maior vulnerabilidade nas condições de saúde, no presente estudo propôs-se a identificar os diagnósticos de enfermagem, segundo a taxonomia II de NANDA, sobre um grupo de idosas consideradas muito pobres. Foram selecionadas 69 idosas, a partir da aplicação do instrumento de Classificação Econômica Brasil (CCEB) em 301 idosos residentes na área de abrangência de um PSF. 0 estado de saúde das idosas foi avaliado utilizando-se a Avaliação Geriátrica Ampla (AGA), que envolve os aspectos funcionais, emocionais, sociais e ambientais. Foram identificadas 23 categorias diagnósticas nas idosas do estudo e uma média de 7,4 diagnósticos/ idosa. Entre os diagnósticos mais freqüentes destacam-se "Mobilidade física prejudicada", "Dor crônica", "Manutenção do lar prejudicada". Os diagnósticos revelaram-se importantes na caracterização das complexas necessidades apresentadas pelas idosas e no grande avanço no direcionamento da assistência.

Palavras-chave: Saúde do Idoso. Programa Saúde da Família. Mulheres. Pobreza.

\begin{abstract}
Considering that the elderly and pauper women are disposed to a larger vulnerability in the health conditions, in the present study it was proposed to identify the nursing diagnosis, according to the taxonomy II of NANDA, about the elderly women group considered very pauper. Were selected 69 elderly women, from the application of the instrument of Brazilian Economic Classification (CCEB) in 301 elderly people that lives in the scope of the FHP. The health condition of the elderly women was evaluated using the Wide Geriatric Evaluation (WGE), that involves the functional, emotional social, and of the environment aspects. Were identified 23 categories of diagnosis in the elderly women of the study and an average of 7.4 diagnosis/elderly. Among the more frequent diagnosis was detached "Damaged Physical Mobility", "Chronicle Pain", "Damaged Maintanance of the Home". The diagnosis revealed to be important in the characterization of the complexity needs presented by the elderly women and in the large advance in the assistance orientation.
\end{abstract}

Keywords:

Elderly's health. Family Health Program. Women. Poverty.

\section{Resumen}

Considerando que las mujeres ancianas pobres son propensas a mayor vulnerabilidad en las condiciones de salud, proponemos en este estudio identificar los diagnósticos de enfermería según la taxonomía II de NANDA de un grupo de ancianas consideradas muy pobres. Fueran seleccionadas 69 ancianas, a partir de la aplicación del instrumento de Clasificación Económica Brasil (CCEB) en 301 ancianos residentes en el área de alcance de un PSF. El estado de salud de las ancianas fue evaluado utilizándose de la Evaluación Geriátrica Amplia (AGA) que involucra los aspectos funcionales, emocionales, sociales y ambientales. Fueron encontradas 23 categorías diagnósticas en las ancianas del estudio y un promedio de 7,4 diagnósticos/anciana. Entre los diagnósticos más frecuentes se destacan: "Mobilidad física perjudicada"; "Dolor crónica"; "Manutención del hogar perjudicada". Los diagnósticos se han revelado importantes en la caracterización de las complexas necesidades presentadas por las ancianas y gran avanzo en lo direccionamiento de la asistencia.

Palabras clave: Salud del Anciano. Programa Salud de la Familia. Mujeres. Pobreza.

'Doutora em Enfermagem, Aluna em Nível de Pós--Doutorado da Universidade Federal de São Paulo e Docente da Disciplina de Enfermagem em Saúde Coletiva da Faculdade de Medicina de Marília. 'Doutor em Saúde Coletiva, Professor Adjunto do Departamento de Medicina Preventiva da Escola Paulista de Medicina da Universidade Federal de São Paulo e orientador do projeto original. ${ }^{3}$ Enfermeira, Mestranda em Saúde Coletiva pela Universidade Estadual de São Paulo e Enfermeira da Estratégia de Saúde da Família na cidade de Marília. ${ }^{4}$ Enfermeira Residente do Programa de Residência Multiprofissional em Saúde da Família da Faculdade de Medicina de Marília. ${ }^{5}$ Enfermeira Bolsista de Treinamento Técnico (TT) da FAPESP. 


\section{INTRODUÇÃO}

Profundas mudanças vêm ocorrendo na estrutura etária da população, caracterizada pela acentuada longevidade, atribuída, em especial, às melhores condições de vida das pessoas, no que se refere ao acesso às novas tecnologias de atendimento à saúde. No Brasil, a população idosa, em 2002, perfazia um total de 14,1 milhões de pessoas, e, para 2025, projeta-se um total de 33,4 milhões. Entre 1950 e 2025, estima-se que a população idosa terá crescido 16 vezes contra cinco vezes a população total ${ }^{1}$.

Nesse contexto, vem aumentando a preocupação com esta parcela da população, pois os idosos apresentam condições específicas que os tornam mais vulneráveis a perdas tanto do ponto de vista funcional como emocional, social e econômico, predispondo-os, principalmente, à presença de múltiplas doenças, baixa auto-estima, depressão, incapacidade para realizações e pobreza, com grandes interferências na qualidade de vida.

Destaca-se que na população idosa há uma predominância de mulheres, e tentativas têm sido feitas para explicar essa diferença. Algumas hipóteses sugerem que os homens têm as mais altas taxas de mortalidade relacionadas à violência, acidentes de trânsito e doenças crônicas. Já as mulheres têm as mais altas taxas de morbidade em quase todas as doenças crônicas não-fatais. Além disso, elas são mais inclinadas a prestar atenção aos sinais e sintomas e a procurar assistência mais freqüentemente que os homens ${ }^{2}$.

Os idosos, pela dificuldade de inserção no mercado de trabalho, constituem uma parcela da população vulnerável ao estado de pobreza, passando a depender significativamente de outras fontes de renda, principalmente da aposentadoria. Além disso, tendem a apresentar maior volatilidade na sua estrutura de gastos decorrentes, quase sempre, do inesperado surgimento de despesas, principalmente com a saúde ${ }^{3}$.

Ressalta-se, ao se considerar a pobreza entre os idosos no Brasil, que, apesar de a aposentadoria representar a garantia de direitos e de inclusão social, apresenta valores que não permitem 0 atendimento satisfatório das necessidades de sobrevivência, principalmente para aqueles que envelhecem com doenças e incapacidades ${ }^{4}$, já que $70 \%$ dos aposentados e pensionistas do Instituto Nacional de Seguro Social (INSS) recebem um salário mínimo por mês ${ }^{5}$.

Ao se considerar que as idosas de baixa condição sócioeconômica podem apresentar maior vulnerabilidade para complexos problemas de saúde, acredita-se que a identificação dos diagnósticos de enfermagem, de acordo com a classificação proposta por NANDA (North American Nursing Diagnoses), poderá trazer contribuições para a melhoria da qualidade da assistência.

A NANDA, contribuindo para o desenvolvimento e refinamento dos diagnósticos de enfermagem, tem desenvolvido um sistema conceitual para classificar os diagnósticos em uma taxonomia, o qual vem sendo constantemente atualizado. Em 1989, publicou a taxonomia I e, com base em diversas avaliações, publicou, em 2001, a taxonomia II, composta por 13 domínios, 106 classes e 155 diagnósticos de enfermagem ${ }^{6}$.
Entre as vantagens dos diagnósticos de enfermagem, destacam-se que eles direcionam os cuidados, fortalecem a atuação profissional nos aspectos relacionados às especificidades da enfermagem, contribuem na identificação de lacunas conceituais, validam as funções da enfermagem e aumentam a autonomia do profissional ${ }^{7,8}$.

Por nossa experiência de utilização dos diagnósticos, segundo a taxonomia da NANDA, podemos afirmar que essa taxonomia representa uma forma de raciocínio lógico que possibilita a inter-relação de causas e efeitos das alterações apresentadas, facilitando o estabelecimento de metas, a adoção de condutas de enfermagem e a realização da avaliação da assistência prestada.

Além disso, a elaboração dos diagnósticos segundo a NANDA exige uma coleta de dados completa, que aborde os múltiplos aspectos que envolvem o estado de saúde do indivíduo, o que estimula melhoria da qualidade dessa etapa do processo de enfermagem, fundamental para o desenvolvimento das demais.

Em estudo de revisão da literatura, em que foram identificadas as vantagens e desvantagens do uso dos diagnósticos de enfermagem, confirma-se que eles representam um instrumento que pode contribuir para a uniformização da linguagem entre os enfermeiros e para a melhoria da qualidade da assistência, direcionando a assistência de enfermagem e possibilitando seu desenvolvimento, além de ser aplicável a diferentes referenciais teóricos utilizados na enfermagem. No entanto, salienta-se a falta de preparo profissional e a necessidade de mudança de postura dos enfermeiros como limites para sua utilização ${ }^{9}$.

0 sistema de classificação dos diagnósticos de enfermagem representa a busca de um novo referencial centrado no conhecimento da ciência de enfermagem. Essa busca vem ocorrendo em ritmo crescente como forma de enfrentar a pressão para definir o corpo de conhecimentos e habilidades essenciais para a práatica de enfermagem ${ }^{10}$.

Considerando que a identificação dos diagnósticos de enfermagem pode contribuir para o conhecimento das características das pessoas que recebem cuidado de enfermagem, direcionar a assistência e possibilitar 0 fortalecimento das especificidades da profissão, propõe-se, para o presente estudo, identificar os diagnósticos de enfermagem, segundo a taxonomia II da NANDA, em um grupo de idosas consideradas muito pobres que vivem na comunidade.

\section{PERCURSO METODOLÓGICO}

Trata-se de um estudo realizado com idosos residentes na área de abrangência de uma Unidade de Saúde da Família do interior paulista. Tal unidade conta atualmente com 1.012 famílias cadastradas, num total de 4.256 pessoas, sendo que destas 461 encontram-se na faixa etária acima dos 60 anos.

A população de referência foi constituída pelo universo de idosos residentes na área citada ( $n=461)$. A identificação deuse por meio do cadastro feito pelos agentes comunitários de saúde. Participaram efetivamente do estudo 301 (65,3\%) idosos; , sendo que, entre os demais, $96(20,8 \%)$ recusaramse a participar, e outros não foram encontrados após a segunda 
visita e/ou eram portadores de doenças mentais e encontravamse sozinhos no domicílio. Dos idosos entrevistados, 186 (61,8\%) são do sexo feminino e 115 (38,2\%), do sexo masculino.

No presente estudo, optou-se por selecionar as mulheres idosas muito pobres, uma vez que tais variáveis indicam maior vulnerabilidade às necessidades de saúde. A seleção delas deu-se a partir da aplicação do instrumento de classificação do estrato social - Critério de Classificação Econômica Brasil (CCEB) proposto pela ANEP (Associação Nacional das Empresas de Pesquisa), aprovado pela ABA (Associação Brasileira Anunciantes) e aceito pela ABIPEME (Associação Brasileira dos Institutos de Mercado).

O CCEB trata-se de um indicador sócio-econômico que visa a estimar o potencial de compra das famílias dos consumidores em áreas urbanas e segmentar o mercado em classes sociais e, apesar de desconsiderar variáveis importantes como a aptidão para o consumo, é considerado um critério objetivo e que evita o constrangimento causado no questionamento da renda pessoal ou familiar ${ }^{11}$. Na sua utilização, atribui-se pontuação a determinados itens em que os indivíiduos se incluemi, e, a partir desta pontuação, são classificados em seis classes (A1, A2, B1, B2, C e D). As.

Segundo tal critério, as pessoas consideradas muito pobres são aquelas pertencentes à classe $\mathrm{D}$;, desta forma, foram selecionadas 69 idosas de tal classe, ou seja, $(37,1 \%)$ do total das mulheres idosas entrevistadas.

Para a coleta de dados das idosas selecionadas, foi utilizado um instrumento elaborado a partir de dados da literatura geriátrica e gerontológica visando à Avaliação Geriátrica Ampla (AGA) ${ }^{12,13,14}$, em que constam os seguintes itens:

1. características sócio-demográficas;

2. lazer, convívio familiar e interação social;

3. hábitos de vida - fumo, álcool, prevenção de câncer de útero/mama e atividade física;

4. número de internação no último ano, estado cognitivo, estado emocional, grau de dependência, pressão arterial no momento da entrevista, avaliação da presença e graduação da dor e doenças referidas.

0 grau de dependência foi verificado a partir de uma escala com 12 indicadores de dependência para o idoso, que considera as atividades instrumentais de vida diária (fazer compras, pegar ônibus, administrar as finanças e tomar os medicamentos adequadamente) e as atividades básicas de vida diária (continência, vestir-se, tomar banho, comer, pentear-se, ir ao banheiro, passar para cama e sair dela), organizadas hierarquicamente. De acordo com as 12 atividades de vida diária citadas, os idosos são classificados em quatro categorias, a saber: a. independente total-idoso capaz de realizar todas as atividades; $b$. dependente leve - idoso que necessita de ajuda para realizar de uma a três atividades de vida diária; $c$. dependente moderado - idoso que necessita de ajuda para realizar de quatro a seis atividades de vida diária; e $d$. dependente severo, aquele que necessita de ajuda para realizar sete e ou mais dessas atividades ${ }^{15}$.

A capacidade cognitiva dos idosos foi avaliada por meio do "Mini exame do estado mental" (MEEN), com a finalidade de caracterizar a presença de prejuízos cognitivos. 0 teste avalia orientação espacial, atenção, capacidade para realização de cálculos simples, memória recente e linguagem. 0 escore final é a soma de todos os pontos obtidos no teste com um valor máximo de 30 pontos. Como é possível, no entanto, haver influência de fatores como o nível de escolaridade, sócio-cultural, alterações perceptivas e de psicopatologias, a nota de corte é 24 para pessoas alfabetizadas e 17 para as não- alfabetizadas ${ }^{13}$.

$\mathrm{Na}$ avaliação do estado emocional, utilizou-se a Escala de Depressão Geriátrica de Yesavage, versão simplificada com 15 perguntas, a qual tem demonstrado confiabilidade na prática clínica ${ }^{11,15}$. Para avaliação da presença e graduação da dor, utilizou-se uma escala unidimensional - Escala Comportamental (EC) - que se baseia no comportamento da pessoa. Ao comportamento álgico é atribuída uma nota, interrogando diretamente a pessoa sobre sua lembrança da dor em função de suas atividades da vida diária ${ }^{14}$.

5. As alterações funcionais foram identificadas a partir de interrogatório sobre os diversos sistemas e aparelhos do corpo humano;

6. Doenças referidas;

7. Adesão ao uso de medicamentos ${ }^{17}$;

8. Os riscos para quedas entre os idosos foram avaliados, considerando-se os principais fatores intrínsecos e extrínsecos $^{7}$. 0 equilíbrio e a marcha, aspectos essenciais em relação a riscos de quedas e mobilidade física, foram verificados com a utilização do instrumento "Performance Oriented Mobility Assessment - POMA" adaptado culturalmente para o Brasil ${ }^{18}$;

9. Na avaliação do estado nutricional, consideraramou-se o índice de massa corporal (peso e altura) e, a distribuição de gordura corporal (circunferência da cintura).

A coleta de dados foi realizada no domicílio dos idosos, por estudantes da segunda e terceira séries dos cursos de Enfermagem e Medicina da Faculdade de Medicina de Marília, que se dispuserem a tal atividade, visto que eles vêm desenvolvendo habilidades e conhecimentos para realização de entrevista e exame clínico desde a primeira série. Para a utilização do instrumento, eles foram capacitados e contaram com acompanhamento e supervisão da pesquisadora responsável.

Para chegar aos diagnósticos de enfermagem, os dados referentes ao estado de saúde das idosas foram submetidos a um processo de raciocínio diagnóstico ${ }^{19}$, o qual é desenvolvido em duas etapas. $\mathrm{Na}$ primeira etapa, os dados passam por análise e síntese.

A fase de análise possibilitou a categorização dos achados, segundo proposta do modelo utilizado para a coleta de dados, e, na seqüência, os dados foram analisados quanto à congruência e lacunas e necessidade de retomada da coleta. No processo de síntese, os dados foram agrupados em padrões por comparação com os conceitos, normas e modelos existentes na literatura geriátrica e gerontológica, levando a hipóteses sobre a situação e ao estabelecimento das causas relacionadas à inferência.

Na segunda fase, os diagnósticos foram estabelecidos de acordo com a taxonomia II da NANDA, incluindo categoria 
diagnóstica, fatores relacionados/fatores de risco e características definidoras.

Os diagnósticos de enfermagem foram elaborados por duas das pesquisadoras, e os registros do raciocínio diagnóstico foram analisados por uma enfermeira do PSF e uma outra da área de geriatria e gerontologia com alguma experiência no ensino e pesquisa de diagnóstico de enfermagem segundo NANDA. No caso de lacunas, as idosas foram revisitadas pelas próprias pesquisadoras, com a finalidade de completar os dados.

Para a realização do estudo, houve autorização do Secretário da Saúde do Município e da equipe de saúde da unidade. Participaram dele os idosos que, após receberem os esclarecimentos, estavam de acordo e assinaram o termo de consentimento livre e esclarecido aprovado pelo comitê de ética e pesquisa com seres humanos da Faculdade de Medicina de Marília.

\section{RESULTADOS E DISCUSSÃO}

A Tabela 1 apresenta as características das idosas, segundo idade, estado civil e escolaridade. Constata-se que a maioria das idosas ésão analfabetas, confirmando os dados analisados pelo IPEA (Instituto de Pesquisa Econômica e Aplicada), que aponta para a forte relação entre pobreza e baixa escolaridade ${ }^{3}$. Além disso, dois terços vivem sem o companheiro. As mulheres idosas que vivem sem o companheiro são altamente vulneráveis àa pobreza e ao isolamento social, o que se deve à discriminação no acesso à educação, salário, alimentação, trabalho significativo, assistência à saúde, medidas de seguro social e poder político ${ }^{20}$.

Tabela 1: Distribuição das idosas segundo idade, escolaridade e estado civil.

\begin{tabular}{|c|c|c|c|c|}
\hline Escolaridade & Estado Civil & $\begin{array}{r}\text { Idad } \\
60-69\end{array}$ & $\begin{array}{l}\text { le }(e m a \\
70-79\end{array}$ & $\begin{array}{l}\text { inos) } \\
80 \text { ou } \\
\text { mais }\end{array}$ \\
\hline Analfabeta & $\begin{array}{l}\text { Sem o companheiro } \\
\text { Com o companheiro }\end{array}$ & $\begin{array}{l}2 \\
3\end{array}$ & $\begin{array}{c}17 \\
8\end{array}$ & $\begin{array}{l}9 \\
2\end{array}$ \\
\hline $1^{\circ} \mathrm{G}$. incompleto & $\begin{array}{l}\text { Sem o companheiro } \\
\text { Com o companheiro }\end{array}$ & $\begin{array}{l}7 \\
5\end{array}$ & $\begin{array}{l}3 \\
6\end{array}$ & $\begin{array}{l}0 \\
3\end{array}$ \\
\hline $1^{\circ} \mathrm{G}$. completo ou mais & $\begin{array}{l}\text { Sem o companheiro } \\
\text { Com o companheiro }\end{array}$ & $\begin{array}{l}3 \\
0\end{array}$ & $\begin{array}{l}0 \\
0\end{array}$ & $\begin{array}{l}0 \\
1\end{array}$ \\
\hline
\end{tabular}

Entre as 69 idosas, foram encontradas 23 categorias diagnósticas e um total de 516 diagnósticos, perfazendo uma média de 7,4 diagnósticos/idosa. Os diagnósticos apresentados são reais e de risco e estão relacionados tanto a aspectos funcionais, como emocionais, sociais e ambientais.

$\mathrm{Na}$ Tabela 2, na página seguinte, encontram-se os diagnósticos de enfermagem presentes em mais de um terço das idosas, bem como os respectivos fatores relacionados a fatores de risco e às características definidoras principais.

0 diagnóstico de "Mobilidade física prejudicada", definido como a "limitação no movimento físico independente e voluntário do corpo ou de uma ou mais extremidades" quase a totalidade das idosas, 65 (94,2\%), demonstra dificuldades relativas à adequada condição de vida, principalmente se considerarmos que se trata de idosas que vivem em condição de pobreza, as quais freqüentemente não dispõem de recursos que possam auxiliá-las na deambulação e mesmo no transporte para que possam viver melhor.

Em estudo realizado com idosos não institucionalizados, foi constatado que $62 \%$ referiram dificuldades para deambular, e, destes, $10 \%$ referiram necessitar de auxílio para caminhar. Acredita-se que os problemas que limitam a deambulação e movimentação precisam ser identificados, avaliados e conduzidos à assistência adequada, para que seja possível manter-lhes a independência ${ }^{21}$.

A "Dor Crônica" representa uma condição presente em 48 $(71,6 \%)$ das idosas, o que revela que elas vivem seu dia-a-dia com algum grau de sofrimento, impedidas de realizar atividades e de interagir com outras pessoas, e até mesmo de manter o padrão de sono e alimentação, considerados básicoas para a sobrevivência.

A dor é uma das queixas mais freqüentes entre os idosos. 0 importante é não subestimar tal queixa, ao considerá-la como parte "normal" do processo de envelhecimento ${ }^{22}$. A alta freqüência de dor em idosos está associada a distúrbioesordens crônicoas, particularmente doenças músculo-esqueléticas como artrites e osteoporoses ${ }^{23}$. A dor é uma das queixas mais freqüentes entre os idosos, sendo importante não subestimar tal fato ao considerá-lo como parte "normal" do processo de envelhecimento ${ }^{22}$.

0 diagnóstico "Manutenção do Lar Prejudicada", presente em 47 (70,1\%) das idosas, é definido como "incapacidade de manter de forma independente um ambiente seguro e que promova o crescimento". Está relacionado à presença de doença, sistemas de apoio inadequados, função cognitiva ou emocional prejudicadas e finanças insuficientes, associandose a acúmulo de sujeira, restos de comida, odores ofensivos, temperatura da casa inadequada (pouco ventilada).

Neste caso, a equipe precisa atuar junto à família e à comunidade com a finalidade de obter ajuda na manutenção de um ambiente confor tável e livre de riscos para a saúde ${ }^{24}$. Além disso, trata-se de uma intervenção que demanda a existência de forte vínculo e conhecimento não só das fragilidades, mas também das potencialidades tanto da família como da comunidade, para que a equipe do PSF possa atuar junto aà uma área adscrita de forma adequada.

"Percepção sensorial visual perturbada", associada principalmente à mudança na capacidade de realizar atividades, esteve presente em 40 (59,7\%) das idosas. Esta condição merece atenção, pois quase sempre é subestimada, sendo considerada tanto pelos familiares como pelo idoso, e até pelos profissionais da saúde, como própria do processo de envelhecimento, sem muita opção de solução. Além disso, para as idosas em condição de pobreza, faltam recursos para aquisição de óculos que poderiam contribuir para melhoria da capacidade visual.

Quase dois terços das idosas, 40 (59,7\%) apresentaram o diagnóstico "Conhecimento deficiente", causado pela falta de exposição, falta de interesse em aprender ou falta de familiaridade com os recursos de informação. Tal condição foi constatada quando as idosas verbalizaram a falta de conhecimento principalmente quanto à doença, uso de medicamento e medidas 
Tabela 2: Distribuição das categorias diagnósticas, principais fatores relacionados/risco e características definidoras.

\section{Diagnóst.}

Fatores Relacionados/Risco
Características Definidoras

Desconforto; dor; falta de conhecimento quanto ao valor Capacidade limitada para desempenhar as habilidades motoras

Mobilidade física prejudicada da atividade física; prejuízos sensorioperceptivos; prejuízos grossas e/ou finas; movimentos não coordenados ou aos músculos-esqueléticos, neuromusculares; intolerância à trancos; amplitude limitada de movimento; mudanças na atividade/força e resistência diminuídas; estado de espírito marcha; velocidade de marcha diminuída, início da marcha depressivo ou ansiedade; estilo de vida sedentário, desuso, dificultado, passos pequenos, pés arrastando-se, oscilação falta de condicionamento; resistência cardiovascular limitada. postural lateral exagerada.

Dor crônica Incapacidade física/psicossocial crônica.

Fadiga; interação reduzida com as pessoas; capacidade alterada de continuar atividades prévias; mudança no padrão de sono.

Doença e lesões; organização ou planejamento familiar Precisam de ajuda para a manutenção da casa; acúmulo de insuficiente; finanças insuficientes; sistemas de apoio sujeira, restos de comida ou resíduos higiênicos; odores inadequado; função cognitiva ou emocional prejudicada.

Manutenção do lar prejudicada

Percepção sensorial Percepção sensorial alterada. visual perturbada

Conhecimento deficiente Interpretação errônea de informação; limitação cognitiva; falta de exposição; falta de interesse no aprender.

Nutrição desequilibrada: mais que as necessidades corporais

Controle ineficaz do regime terapêutico

Dificuldades econômicas; complexidade do regime terapêutico e déficit de suporte social. ofensivos; temperatura da casa inadequada.

Mudança na resposta usual aos estímulos; Mudança na 41 capacidade de resolução dos problemas.

Verbalização do problema; seguimento não acurado de instruções.

Peso $20 \%$ acima do ideal para a altura e compleição; dobra de prega do tríceps, acesso de $15 \mathrm{~mm}$; e nível de atividade sedentário.

Verbaliza desejo de controlar o tratamento da doença e a prevenção de seqüelas; verbaliza dificuldade com a regulação/

Risco para solidão Isolamento social; privação afetiva; falta de energia. interação de um ou mais dos regimes prescritos para a prevenção de complicações e o tratamento da doença ou de seus efeitos; verbaliza que não agiu para incluir os regimes de tratamento nas rotinas diárias.

História de quedas; idade de 65 anos; mulher; mora sozinha; uso de artefatos de auxílio (bengalas); dificuldades visuais e auditivas; artrite; hipotensão ortostática; ausência do Risco para quedas sono; mobilidade física prejudicada, equilíbrio prejudicado, dificuldade na marcha; ambiente com móveis e objetos em excesso; ausência de material antiderrapante em banheiros, escadas e tapetes; uso de medicamentos.

Tristeza crônica

Padrão de sono perturbado

Dentição prejudicada
Doença e deficiência; morte de pessoa amada e crise Depressão; solidão; desesperança; baixa auto-estima; raiva relacionada ao estágio de desenvolvimento.

Pensamentos ruminantes pré-sono; higiene de sono Insônia de manutenção do sono; queixas verbais de inadequada; depressão;solidão; tristeza; medo; ansiedade; dificuldades para adormecer; queixas verbais de não se medicações.

sentir bem descansado; insatisfação com o sono.

Higiene oral ineficaz; barreiras econômicas ou de acesso ao Presença de cáries; perda de dentes; dentes desgastados cuidado profissional; falta de conhecimento a respeito da ou estragados; ausência parcial ou completa de dentes. saúde dental. de promoção da qualidade de vida ou comportamento apático, quando alegaram "já estarem velhao s demais para aprender" ou que "com esta idade não adianta querer muita coisa".

0 conhecimento referente às práticas de saúde tem grande relação com a adesão a elas ${ }^{25}$. Nessa fase da vida, em que aumentam os riscos de agravamento das condições de saúde, é importante que as pessoas consigam se apropriar-se dos conhecimentos necessários à manutenção e melhoria das condições de saúde.

Estudo sobre saberes e práticas adotados pelas mulheres idosas, sujeitos de ações de educação em saúde, mostrou que elas são capazes de aprender e agir, conquistando significado para a sociedade e exercendo plenamente a cidadadania26.

Apesar da condição de pobreza das idosas, 29 (43,3\%) apresentam o diagnóstico de "Nutrição desequilibrada: mais que as necessidades corporais", que pode ser caracterizado pelo peso $20 \%$ ou mais acima do peso ideal para a altura, sedentarismo e dobra da prega do tríceps acima de $25 \mathrm{mmHg}$.

Os indicadores do estado nutricional são considerados importantes preditores de morbi-mortalidade, e dados de uma população adulta de baixa renda também revelam alta incidência de sobrepeso e obesidade, principalmente entre as mulheres ${ }^{27}$.

"Controle ineficaz do regime terapêutico" foi encontrado em $29(43,3 \%)$ das idosas, relacionado, na maioria das vezes, à complexidade do regime terapêutico, dificuldades econômicas e déficit de suporte social. As idosas de baixo poder aquisitivo, além das dificuldades próprias do processo de envelhecimento, enfrentam outros problemas advindos da condição de pobreza, levando a riscos para a atual situação de saúde.

Entre as idosas, a privação afetiva, o isolamento social e a falta de energia levaram a confirmar o diagnóstico de "Risco 
para solidão" em 25 (37,3\%) delas. A condição de fragilidade do idoso, a perda da autonomia e da independência, levam ao esfriamento dos vínculos afetivos. Isso pode estar relacionado a circunstâncias objetivas, como o afastamento do grupo de relações, e subjetivas, a depender da forma como o idoso enfrenta a situação ${ }^{28}$. Em outros grandes centros urbanos, têm aumentado as pequenas famílias em detrimento das famílias extensas, reduzindo a possibilidade de apoio e atenção ao idoso.

0 diagnóstico "Risco de quedas", presente entre as idosas, deve-se a fatores intrínsecos, como dificuldade visual, ausência de sono, dificuldade na marcha e equilíbrio prejudicado, uso de medicação ansiolítica, antidepressiva e anti-hipertensiva, e a fatores extrínsecos, entre eles, a presença de escadas e piso escorregadios, tapetes soltos e uso de calçado inapropriado. Entre os fatores que têm contribuído para agravar as condições de saúde e de vida da população idosa destacam-se as quedas, pois constituem a primeira causa de acidentes em pessoas com mais de 60 anos $^{29}$. Essas, no entanto, são passíveis de prevenção, havendo a necessidade de ações educativas envolvendo tanto a idosa como os familiares.

Ao longo da vida, as pessoas idosas estão propensas a múltiplas perdas, seja pela presença de doenças e incapacidades, morte ou ausência de pessoas que lhes são próximas, o que quase sempre desencadeia tristeza progressiva e recorrente. A condição de "Tristeza crônica" foi encontrada em 26 (37,6\%) das idosas, e foi expressa como depressão, solidão, desesperança, baixa auto-estima, raiva e frustração.

Das idosas, 23 (34,3\%) apresentaram o diagnóstico de "Padrão de sono perturbado", relacionado, na maioria das vezes, a ansiedade, higiene do sono inadequado, tristeza, entre outros, que se associam tanto à qualidade como à quantidade do sono. Os transtornos do sono, considerados freqüentes entre os idosos, afetam suas relações e o desempenho das atividades da vida diária, com grande interferência na sua qualidade de vida.

Das idosas, 22 (32,8\%) apresentaram "Dentição prejudicada" associada à ausência de alguns dentes ou de todos eles, dentes desgastados, cáries, além do uso de prótese mal adaptada. Ao referir-se ao atual quadro em que se encontra a saúde bucal dos idosos, é necessário considerar que esse grupo de pessoas carrega a herança de práticas de saúde curativas e mutiladoras ${ }^{30}$. Além disso, a falta de acesso da população aos serviços de saúde bucal é outro aspecto importante a ser considerado. 0 impacto da saúde bucal para a qualidade de vida na terceira idade revela-se no aspecto funcional, social e psicológico ${ }^{31}$.

Outros diagnósticos menos freqüentes entre as idosas foram: "Constipação" presente em 15 (21,8\%) idosas; "Percepção sensorial auditiva perturbada" em 12 (17,4\%) idosas; "Fadiga" em 10 (14,5\%) idosas; "Processos familiares interrompidos" em cinco (7,3\%) idosas; "Nutrição desequilibrada: menos que as necessidades corporais" e "Manutenção ineficaz da saúde" em 4quatro (5,8\%) idosas; "Deglutição prejudicada" em três $(4,4 \%)$ idosas e "Incontinência urinária funcional" em duas (2,9\%) idosas. Os diagnósticos: "Percepção sensorial olfativa perturbada", "Comunicação verbal prejudicada"; "Memória prejudicada" apareceram, cada um deles, apenas em uma idosa (1,5\%).
Alguns diagnósticos, como é o caso da "Mobilidade física prejudicada", "Dor crônica"e "Fadiga", comprometem as atividades de recreação e predispõem as idosas à inatividade, podendo levar aos diagnósticos de "Atividade de recreação deficiente" e de "Risco de intolerância à atividade". No entanto, considerou-se que os três primeiros diagnósticos seriam prioritários e que, ao se traçarem estratégias de intervenção para eles, seria possível a melhoria das condições relacionadas aos outros dois.

\section{CONSIDERAÇÕES FINAIS}

No presente estudo, propôs-se caracterizar as necessidades de cuidados a partir da identificação dos diagnósticos de enfermagem segundo NANDA, de mulheres idosas consideradas muito pobres de uma área adscrita, uma vez que tais condições indicam maior vulnerabilidade no estado de saúde. As 69 idosas estudadas apresentaram 23 categorias diagnósticas, com uma média de 7,4 diagnósticos/idosa. Entre os diagnósticos mais freqüentes destacam-se "Mobilidade física prejudicada", "dor crônica", "Manutenção do lar prejudicada", "Percepção sensorial visual perturbada", "Conhecimento deficiente" e "Nutrição desequilibrada: mais que as necessidades corporais". Tais diagnósticos revelam que as idosas demandam intervenções nos aspectos sociais, funcionais, emocionais e ambientais. Além disso, considera-se que eles são de grande utilidade para caracterizar as necessidades de cuidado de uma população, permitindo intervenções individuais e em grupos.

As características definidas e os fatores relacionados contribuem para maior compreensão das possibilidades de intervenção e para o direcionamento do cuidado, uma vez que permite a especificação das respectivas causas e manifestações da necessidade apresentada.

Além de os diagnósticos de enfermagem indicarem as especificidades da profissão, dão a dimensão da complexidade que a denominada "rede básica" ou "atenção primária" está trabalhando atualmente, tendo em vista o envelhecimento da população.

Constata-se, então, que, para atuar junto a essa parcela da população, é necessário pautar-se na integralidade do cuidado e na lógica da vigilância da saúde visando à promoção, prevenção, cura e reabilitação das condições de saúde. Neste contexto, o papel do enfermeiro assume um dimensionamento ampliado e, muitas vezes, distinto das bases de formação e de atuação dos profissionais de saúde que, por muitos anos, vem privilegiando 0 tecnicismo e deixando margens pouco definidas e, portanto, conflituosas ao implementar o trabalho interdisciplinar.

Concordamos quando se afirma: "à medida que continuam os esforços para desenvolver terminologias de referência que compreendam a totalidade da experiência de assistência à saúde, a Eenfermagem deve posicionar-se como parte integrante desse desenvolvimento e deve certificar-se de que está claramente representada nessas terminologias" 7 .

Atuar nesta lógica, além de ser um desafio para a Eenfermagem brasileira nos dias atuais, representa também, um grande avanço para a profissão enquanto integrante de uma equipe multiprofissional, considerando a clara necessidade de delimitação do seu papel diante dfrente ao cuidado das pessoas, famílias e comunidade. 


\section{Referências}

1. Garrido R, Menezes PR. O Brasil está envelhecendo: boas e más noticias por uma perspectiva metodológica. Rev Bras Psiquiatr 2002 abr; 24 (supl 1): 3-6.

2. Fundação Sistema Estadual de Análise de Dados-SEAD (SP). Esperança de vida aumenta e diferença entre gêneros diminui: queda de homicídios em jovens poupa vidas e explica avanço masculino. [on-line]. 2007. [citado 31 maio 2007]. Disponível em: http:// www.seade.gov.br/produtos/espvida/espvida_jan2006.pdf.

3. Barros RP, Mendonça R, Santos D. Incidência e natureza da pobreza entre idosos do Brasil. Textos para discussão [periódico on-line] 1999 dez; [citado 17 jul 2007]. [aprox 32 telas]. Disponível em: http://www.ipea.gov.br/pub/td/td0686.pdf.

4. Fernandez MGM, Santos SR. Políticas públicas e direitos do idoso: desafios da agenda social do Brasil contemporâneo. Achegas.net: Rev Cienc Politica [periódico on-line] 2007 mar/abr; [citado 18 jul 2007], (34): [aprox 12 telas]. Disponível em: http://www.achegas.net/ numero/34/idoso_34.pdf.

5. Goldman SN. As dimensões sociopoliticas do envelhecimento. In: Py L, Pacheco JL, Sá JLM, Goldman SN, organizadores. Tempo de envelhecer: percursos e dimensões psiocossiais. Rio de Janeiro (RJ): Nau; 2004. p. 61-81.

6. Braga CG, Cruz DALM. A taxonomia II proposta pela North American Nursing Diagnoses Association-NANDA. Rev Latino-am Enfermagem 2003 mar/abr; 11(2): 240-44.

7. North American Diagnosis Association - NANDA. Diagnósticos de enfermagem da NANDA: definições e classificação: 2001-2002. Porto Alegre (RS): Artmed; 2004.

8. Carvalho EC. A utilização do diagnóstico de enfermagem: experiência no ensino. Anais do $1^{\circ}$ Simpósio Internacional sobre Diagnóstico de Enfermagem; 1995; João Pessoa (PB), Brasil. João Pessoa (PB); 1995. p. 81-93.

9. Marin MJS, Messias DCC, Ostroski LE. Análise das publicações sobre diagnóstico de enfermagem no Brasil. Nurs, São Paulo, 2004 set; 7(76): 23-8.

10. Cruz DALM. A inserção do diagnóstico de enfermagem no processo assistencial. In: Cianciarullo TI, Gualda DMR, Melleiro MM, Anabuki MH, editores. Sistema de assistência de enfermagem: evolução e tendências. São Paulo(SP): Ícone; 2001. p.63-84.

11. Mattar FN. Análise crítica dos estudos de estratificação socioeconômica de ABA-Abipeme. Rev Adm 1995 jan/mar; 30(1): 57-74.

12. Almeida OP, Almeida SA. Confiabilidade da versão brasileira da Escala de Depressão em Geriatria-GDS (versão reduzida). Arq Neuropsiquiatr 1999 jun; 57(2B): 421-26.

13. Brucki SMD, Nitrini R, Caramelli P, Bertolucci PHF, Okanoto IH. Sugestões para o uso do mini-exame do estado mental no Brasil. Arq Neuropsiquiatr 2003 set; 61(3B): 777-81.

14. Saúde em movimento. Dor: mensuração [homepage na Internet]. 20002001 [citado 29 maio 2007]; [aprox 1 tela]. Disponível em: http:// unw.saudeemmovimento.com.br/conteudos/conteudo_exibe1.asp?cod_noticia=39

15. Ramos LR, Perracini MR, Rosa TE, Kalache A. Significance and management of disability amoung urban elderly residents in Brazil. J Cross Cult Gerontol 1993; 8: 313-23.
16. Oliveira DAAP, Gomes L, Oliveira, RF. Prevalência de depressão em idosos que freqüentam centros de convivência. Rev Saude Publica 2006 ago; 40(4): 734-36.

17. Burguez Diaz R. Adesão ao tratamento medicamentoso em pacientes idosos. In: Papaléo Neto M. Gerontologia: a velhice e o envelhecimento em visão globalizada. São Paulo (SP): Atheneu; 1996. p. 230-41.

18. Gomes GC. Tradução, adaptação transcultural e exame das propriedades de medida da escala "Performance-Oriented Mobility Assessment- POMA- para uma amostragem de idosos brasileiros institucionalizados [dissertação de mestrado]. Campinas (SP): Universidade Estadual de Campinas; 2003.

19. Risner PB. Diagnosis analysis and synthesis of date. In: Christensen PJ, Kenney JW, editors. Nursing process: application of conceptual models. St. Louis (USA): Mosby; 1990. p. 132-57.

20. World Health Organization-WHO. Envelhecimento ativo: uma política de saúde. [monografia on-line]. Brasília (DF): OPAS; 2005.

21. Campedelli MC, Rotter MV, Silva MR. Deambulação: uma problemática no paciente idoso. Ambito Hosp 1992 jan; 3(34): 3-12.

22. Anzola Perez E, Galinsky D, Morales Martines F, Salas AR, Sánchez Ayondez M. La atención de los ancianos: um desafio para los años noventa. Washington (USA): Organización Panamericana de la Salud; 1994.

23. Andrade FA, Pereira LV, Sousa FAEF. Mensuração da dor no idoso: uma revisão. Rev Latino-am Enfermagem 2006 mar/abr; 14(2): 271-76.

24. Carpenito $\sqcup$. Manual de diagnóstico de enfermagem. $9^{a}$ ed. Porto Alegre (RS): Artmed; 2003.

25. Silveira LMC, Ribeiro VMB. Grupo de adesão ao tratamento: espaço de "ensinagem" para profissionais de saúde e pacientes. Interface Comun Saude Educ 2004/2005; 9(16): 91-104.

26. Monteiro CFS, Luz MHA. Educação em saúde e mulheres idosas: promoção de conquistas políticas, sociais e em saúde. Esc Anna Nery Enferm 2006 dez; 10(3): 456-61.

27. Anjos LA, Silva DO, Serrão AS, Silva CVC. Vigilância nutricional em adultos: experiência de uma unidade de saúde atendendo população favelada. Cad Saude Publica 1992 jan/mar; 8(1): 50-6.

28. Heredia VBM, Cortelletti IA, Casara MB. Abandono na velhice. Textos sobre Envelhecimento 2005; 8(3): 1-11.

29. Yamaguchi AM. Quedas na terceira idade [on- line] 2000. [citado 10 jul 2007]. Disponível em: http://www.saudetotal.com/artigos/idoso/quedas.asp

30. Moreira RS, Nico LS, Tomita NE, Ruiz T. A saúde bucal do idoso brasileiro: revisão sistemática sobre o quadro epidemiológico e acesso aos serviços de saúde bucal. Cad Saude Publica 2005 nov/dez; 21 (6): 1665-675.

31. Shinkai RSA, Cury AADB. 0 papel da odontologia na equipe interdisciplinar: contribuindo para a atenção integral ao idoso. Cad Saude Publica 2002 out/dez; 16(4): 1099-109.

\section{Nota}

aParte do projeto "Os idosos precisam de cuidados especiais: uma proposta de planejamento em PSF", financiado pela FAPESP com Parecer $n^{0}$ 06/54664-3. 